\title{
A REMARK ON HÖRMANDER'S UNIQUENESS THEOREM
}

\author{
D. DEL SANTO AND X. SAINT RAYMOND
}

(Communicated by Barbara L. Keyfitz)

\begin{abstract}
By using the paradifferential calculus, Hörmander's classical uniqueness theorem for the Cauchy problem is shown to hold for operators with $\mathscr{C}^{2}$ coefficients in the principal part, instead of $\mathscr{C} \infty$, under a special normality assumption.
\end{abstract}

This work is devoted to the local uniqueness of the solutions to the Cauchy problem for partial differential operators.

This subject has been studied widely during the last ten years, and we refer to $[1,7]$ for a comprehensive bibliography.

Our main aim is to give a partial answer to a question asked by Hörmander [5, Chapter XXVIII], specifically, after having proved his uniqueness theorem for principally normal operators with $\mathscr{C}^{\infty}$ coefficients in the principal part, under a convexity condition, Hörmander remarks that it is not clear how smooth the coefficients must be for this theorem to hold.

We think that the requirement on the smoothness of the coefficients in the principal part essentially depends on the strength of the normality assumption. Indeed, it was already observed by Hörmander [4, Chapter 8 ] that $\mathscr{C}^{1}$ coefficients are sufficient in the case of elliptic or real principal part.

In this note, we show that the assumption of $\mathscr{C}^{2}$ coefficients will work under a strong normality assumption.

In order to give a precise statement, let us set the problem. Let $\Omega$ be an open set of $\mathbf{R}^{n}$ and let $P(x, D)$ be the operator

$$
P(x, D)=\sum_{|\alpha| \leq m} a_{\alpha}(x) D^{\alpha}
$$

with $a_{\alpha} \in \mathscr{C}^{2}(\Omega, \mathbf{C})$ for $|\alpha|=m, a_{\alpha} \in L_{\text {loc }}^{\infty}(\Omega, \mathbf{C})$ for $|\alpha| \leq m-1$. Let $S$ be a hypersurface in $\Omega, S=\{x \in \Omega \mid \varphi(x)=0\}$ where $\varphi \in \mathscr{C}^{2}(\Omega, \mathbf{R})$, and let $x_{0} \in S$ such that $\varphi^{\prime}\left(x_{0}\right) \neq 0$. For our specific purposes, we rewrite Definitions 28.2.4 and 28.3.1 of [5] in the following equivalent forms.

Definition 1. We say that $P$ is principally normal at $x_{0}$ if there exist a neighborhood $V$ of $x_{0}$ and a function $q \in L_{\text {loc }}^{\infty}\left(T^{\star} V \backslash 0\right)$ such that $q(x, \xi)$ is

Received by the editors June 24, 1991.

1991 Mathematics Subject Classification. Primary 35A07, 35B45, 35 S50. 
homogeneous in $\xi$ of degree $m-1$ and

$$
\{\bar{p}, p\}(x, \xi)=2 i \operatorname{Re}(\bar{q}(x, \xi) p(x, \xi)) \text { for all }(x, \xi) \in T^{\star} V \backslash 0 . \dagger
$$

Definition 2. We say that the half-space $S^{+}=\{x \in \Omega \mid \varphi(x)>0\}$ is strongly pseudoconvex with respect to $P$ at $x_{0}$ if for each $\zeta=\xi-i \tau \varphi^{\prime}\left(x_{0}\right) \neq 0, \xi \in \mathbf{R}^{n}$, $\tau \in \mathbf{R}$, such that $p\left(x_{0}, \zeta\right)=\{p, \varphi\}\left(x_{0}, \zeta\right)=0$ we have

$$
\begin{aligned}
\frac{1}{2 i \tau}\left\{\overline{\tau_{\tau \varphi}}, p_{\tau \varphi}\right\}\left(x_{0}, \xi\right)>0 & \text { if } \tau \neq 0, \\
\operatorname{Re}\{\bar{p},\{p, \varphi\}\}\left(x_{0}, \xi\right)<0 & \text { if } \tau=0
\end{aligned}
$$

(here $\left.p_{\tau \varphi}(x, \xi)=p\left(x, \xi-i \tau \varphi^{\prime}(x)\right)\right)$.

We can now state the main result.

Theorem. Assume that the differential operator $P(x, D)$ has $\mathscr{C}^{2}$ coefficients in the principal part and bounded coefficients in the lower order terms. Assume that its principal symbol $p(x, \xi)$ satisfies condition (2) for a function $q(x, \xi)$ of class $\mathscr{C}^{1}$ in $x$ and $\mathscr{C}^{\infty}$ in $\xi$, $\ddagger$ homogeneous in $\xi$ of degree $m-1$, and that the half-space $S^{+}$is strongly pseudoconvex with respect to $P$ at $x_{0}$.

Then the following uniqueness property holds: for any $u \in H_{\mathrm{loc}}^{m-1}\left(\mathbf{R}^{n}\right)$ such that $P(x, D) u=0$ and $u_{\mid \varphi<0} \equiv 0$ near $x_{0}$, we have $u \equiv 0$ near $x_{0}$.

Remark 1 . The normality condition in the Theorem will be satisfied if it can be shown that an equality $\{\bar{p}, p\}=2 i \operatorname{Re}(\bar{q} p)$ holds near every zero of $p$ for some local function $q$; indeed, a global $q$ can then be constructed by using a partition of unity.

Remark 2. Our result contains the first statement of Hörmander's uniqueness theorem [4, Theorem 8.9.1], without the requirement that $q$ is a polynomial in $\xi$.

Remark 3. We could also give a result under the weaker assumption that $P$ is principally normal (i.e., $q$ is merely locally bounded; see Definition 1 ) if we were able to prove a Fefferman-Phong inequality for paradifferential operators, as it is easily seen in the proof below.

As in the model [5, Theorem 28.3.4] the uniqueness result follows from a Carleman estimate proved for a weight function admitting a given family of smooth strongly pseudoconvex level surfaces (see [5, Proposition 28.3.3]). Thus, if this family is given as the level surfaces of a function $\psi$, then we may assume, after a change of variables, that $\psi(x)=x_{n}$; then we set $\phi(x)=-e^{-A \psi(x)} \quad(\phi$ is then an increasing negative function of $x_{n}$ ). Introducing the norms

$$
\|u\|_{s, \lambda}^{2}=(2 \pi)^{-n} \int\left(\lambda^{2}+|\xi|^{2}\right)^{s}|\hat{u}(\xi)|^{2} d \xi
$$

and the neighborhoods of $x_{0}, \Omega_{\varepsilon}=\left\{x \in \Omega|| x-x_{0} \mid<\varepsilon\right\}$, the result stated in the Theorem can be classically deduced from the following estimate.

$\dagger p(x, \xi)=\sum_{|\alpha|=m} a_{\alpha}(x) \xi^{\alpha}$ is the principal symbol of operator $P$ and $\{\cdot, \cdot\}$ are the usual Poisson brackets.

$\ddagger$ This means, as usual, that $\partial_{\xi}^{\alpha} q$ is a $\mathscr{C}^{1}$ function of $(x, \xi)$ for all $\alpha \in \mathbf{Z}_{+}^{n}$. 
Proposition. Let $P$ be as in the statement of our theorem and assume that the half-spaces $\{x \in \Omega \mid \psi(x)>c\}, \psi(x)=x_{n}, c \in \mathbf{R}$, are strongly pseudoconvex in a neighborhood of $x_{0}$.

Then there exist constants $A, C$, and $\varepsilon>0$ such that, with $\phi=-e^{-A \psi}$,

$$
\lambda^{1 / 2}\left\|e^{-\lambda \phi} u\right\|_{m-1, \lambda} \leq C\left\|e^{-\lambda \phi} p(x, D) u\right\|_{0}
$$

for all $u \in \mathscr{C}_{0}^{\infty}\left(\Omega_{\varepsilon}\right)$ and $\lambda \geq 1 / \varepsilon$.

Proof. As usual we set $v=e^{-\lambda \phi} u$ and consider the operator

$$
P_{\lambda}=e^{-\lambda \phi} \circ p(x, D) \circ e^{\lambda \phi}=p_{\lambda}(x, D)+r_{\lambda}(x, D),
$$

where $p_{\lambda}(x, \xi)=p_{\lambda \phi}(x, \xi)=p\left(x, \xi-i \lambda \phi^{\prime}(x)\right)$ and $r_{\lambda}(x, \xi)$ is an $(m-1)$ st degree polynomial in $(\lambda, \xi)$. With these notations, $(3)$ becomes

$$
\lambda^{1 / 2}\|v\|_{m-1, \lambda} \leq C\left\|P_{\lambda} v\right\|_{0}
$$

for all $v \in \mathscr{C}_{0}^{\infty}\left(\Omega_{\varepsilon}\right)$ and $\lambda \geq 1 / \varepsilon$.

At this point we will use a paradifferential calculus with a large parameter as given in [6, Appendix B], that is, we will use the spaces $\Gamma_{\rho}^{m}$, defined by [6, (B.1.3)], and their properties. Here, it is clear by homogeneity that $p_{\lambda} \in \Gamma_{2}^{m}$. In the proof of (4) we will use the following lemmas.

Lemma 1. Under the assumptions of our proposition, there exist two constants $A$ and $\delta>0$ and a symbol $e_{\lambda} \in \Gamma_{1}^{2 m-2}$ such that for $x$ sufficiently close to $x_{0}$ and $\zeta=\xi-i \lambda \phi^{\prime}(x)$,

$$
\frac{1}{i}\left\{\overline{p_{\lambda}}, p_{\lambda}\right\}-2 \operatorname{Re}\left[\overline{\left(q+\lambda A^{2} \phi p_{\lambda} /|\zeta|^{2}\right)} p_{\lambda}\right]=\lambda e_{\lambda}, \quad e_{\lambda}(x, \xi) \geq \delta\left(\lambda^{2}+|\xi|^{2}\right)^{m-1}
$$

Proof. Let us set $\tau=-\lambda A \phi(x)$ so that $\zeta=\xi-i \lambda \phi^{\prime}(x)=\xi-i \tau \psi^{\prime}(x)$. Recalling that $\psi^{\prime \prime} \equiv 0$, an easy computation gives

$$
\frac{1}{i}\left\{\overline{p_{\lambda}}, p_{\lambda}\right\}=2 \operatorname{Im}\left\langle\overline{p_{\xi}^{\prime}(x, \zeta)}, p_{x}^{\prime}(x, \zeta)\right\rangle+2 A \tau|\{p, \psi\}(x, \zeta)|^{2}
$$

On the other hand, our normality assumption shows that the polynomial in $\tau$

$$
\operatorname{Im}\left\langle\overline{p_{\xi}^{\prime}(x, \zeta)}, p_{x}^{\prime}(x, \zeta)\right\rangle-\operatorname{Re}(\bar{q}(x, \xi) p(x, \zeta))
$$

vanishes for $\tau=0$ and, therefore, we can write $\tau l(x, \xi, \tau)$ where $l$ is a $\mathscr{C}^{1}$ function in $x, \mathscr{C}^{\infty}$ and homogeneous in $(\xi, \tau)$ of degree $2 m-2$. Furthermore, the convexity assumption implies that for $x$ in a compact neighborhood of $x_{0}$, inf $|\zeta|=1\left[l(x, \xi, \tau)+A\left(|\{p, \psi\}(x, \zeta)|^{2}+|p(x, \zeta)|^{2}\right)\right]=\delta>0$ for some large constant $A$. Thus we can use homogeneity to write

$$
\begin{aligned}
\frac{1}{i}\left\{\overline{p_{\lambda}}, p_{\lambda}\right\}-2 \operatorname{Re}\left[\overline{\left(q+\lambda A^{2} \phi \frac{p_{\lambda}}{|\zeta|^{2}}\right)} p_{\lambda}\right] \\
=2 \tau l(x, \xi, \tau)+2 A \tau|\{p, \psi\}(x, \zeta)|^{2}+2 A \tau \frac{|p(x, \zeta)|^{2}}{|\zeta|^{2}} \\
=2 \tau|\zeta|^{2 m-2}\left[l\left(x, \frac{\xi}{|\zeta|}, \frac{\tau}{|\zeta|}\right)\right. \\
\left.\quad+A\left(\left|\{p, \psi\}\left(x, \frac{\zeta}{|\zeta|}\right)\right|^{2}+\left|p\left(x, \frac{\zeta}{|\zeta|}\right)\right|^{2}\right)\right] \\
=\lambda e_{\lambda}(x, \xi)
\end{aligned}
$$

where $e_{\lambda}$ has the properties stated in Lemma 1 . 
Lemma 2. With the notation of Lemma 1, the symbol

$$
\tilde{p}_{\lambda}=p_{\lambda}+q+\lambda A^{2} \phi \frac{p_{\lambda}}{|\zeta|^{2}}
$$

satisfies $\tilde{p}_{\lambda} \in \Gamma_{2}^{m}+\Gamma_{1}^{m-1}$. Moreover, the operator

$$
R_{\lambda}=\left(T_{p_{\lambda}}^{\lambda}\right)^{*} \circ T_{p_{\lambda}}^{\lambda}-T_{\tilde{p}_{\lambda}}^{\lambda} \circ\left(T_{\tilde{p}_{\lambda}}^{\lambda}\right)^{*}-\lambda T_{e_{\lambda}}^{\lambda}
$$

is bounded from $H^{m-1}$ into $H^{1-m}$ uniformly in $\lambda$ (for the definition of $T_{p_{\lambda}}^{\lambda}$ see [6, (B.1.7)]).

Proof. The property $\tilde{p}_{\lambda} \in \Gamma_{2}^{m}+\Gamma_{1}^{m-1}$ simply follows from the fact that $\tilde{p}_{\lambda}$ is a sum of homogeneous functions of $(\xi, \lambda)$. As for the boundedness of $R_{\lambda}$, we continue to use that all our symbols are sums of homogeneous terms, so that it is an easy consequence of the following result that refines [6, Proposition B.1.3], but the proof of which is a straightforward extension of the classical paradifferential machinery (see [2, Theorem 3.9; 3, §3]).

Lemma 3. Let $\rho, \sigma, m$, and $l$ be positive integers and set, for homogeneous symbols $a \in \Gamma_{\rho}^{m}$ and $b \in \Gamma_{\sigma}^{\prime}$,

$$
a^{*}=\sum_{|\alpha|<\rho} \frac{1}{\alpha !} \partial_{\xi}^{\alpha} D_{x}^{\alpha} \bar{a} \quad \text { and } a \# b=\sum_{|\alpha|<\sigma} \frac{1}{\alpha !} \partial_{\xi}^{\alpha} a D_{x}^{\alpha} b .
$$

Then $\left(T_{a}^{\lambda}\right)^{*}-T_{a^{*}}^{\lambda}$ (respectively, $\left.T_{a}^{\lambda} \circ T_{b}^{\lambda}-T_{a \# b}^{\lambda}\right)$ is bounded from $H^{s}$ into $H^{s-m+\rho}$ (respectively into $H^{s-m-l+\sigma}$ ) uniformly in $\lambda$.

Proof of estimate (4). First, we can write

$$
\begin{aligned}
\left\|P_{\lambda} v\right\|_{0} & \geq\left\|p_{\lambda}(x, D) v\right\|_{0}-\left\|r_{\lambda}(x, D) v\right\|_{0} \\
& \geq\left\|T_{p^{\lambda}}^{\lambda} v\right\|_{0}-\left\|\left(p_{\lambda}(x, D)-T_{p_{\lambda}}^{\lambda}\right) v\right\|_{0}-\left\|r_{\lambda}(x, D) v\right\|_{0} \\
& \geq\left\|T_{p_{\lambda}}^{\lambda} v\right\|_{0}-C\|v\|_{m-1, \lambda},
\end{aligned}
$$

thanks to estimates of [6, Proposition B.1.2]. On the other hand, we have

$$
\left\|T_{p_{\lambda}}^{\lambda} v\right\|_{0}^{2}=\left(R_{\lambda} v, v\right)+\left\|\left(T_{\tilde{p}_{\lambda}}^{\lambda}\right)^{*} v\right\|_{0}^{2}+\lambda\left(T_{e_{\lambda}}^{\lambda} v, v\right) \geq \lambda\left(T_{e_{\lambda}}^{\lambda} v, v\right)-C\|v\|_{m-1, \lambda}^{2},
$$

thanks to the uniform boundedness of $R_{\lambda}$ given by Lemma 2 . Finally the estimate we have for $e_{\lambda}$ in Lemma 1 and a Gårding inequality such as in [6, Proposition B.1.4] imply,

$$
\left\|T_{p_{\lambda}}^{\lambda} v\right\|_{0}^{2} \geq \frac{\delta}{2} \lambda\|v\|_{m-1, \lambda}^{2}-C \lambda\|v\|_{m-2, \lambda}^{2}-C\|v\|_{m-1, \lambda}^{2},
$$

whence we get (4) if $\varepsilon$ is chosen sufficiently small and $\lambda$ sufficiently large to absorb all the error terms in the main term $(\delta / 2) \lambda\|v\|_{m-1, \lambda}^{2}$.

\section{REFERENCES}

1. S. Alinhac, Uniqueness and non-uniqueness in the Cauchy problem, Contemp. Math., vol. 27, Amer. Math. Soc., Providence, RI, 1984, pp. 1-22.

2. J.-M. Bony, Calcul symbolique et propagation des singularités pour les équations aux dérivées partielles non-linéaires, Ann. Sci. École. Norm. Sup. (4) 14 (1981), 209-246. 
3. P. Gérard and J. Rauch, Propagation de la régularité locale de solutions d'équations hyperboliques non-linéaires, Ann. Inst. Fourier (Grenoble) 37 (1987), 65-84.

4. L. Hörmander, Linear partial differential operators, Springer-Verlag, Berlin, 1963.

5. _ The analysis of linear partial differential operators, vol. IV, Springer-Verlag, Berlin, 1985.

6. G. Métivier, Interaction de deux chocs pour un système de deux lois de conservation, en dimension deux d'espace, Trans. Amer. Math. Soc. 296 (1986), 431-479.

7. C. Zuily, Uniqueness and non-uniqueness in the Cauchy problem, Progr. Math., vol. 33, Birkhäuser, Boston, MA, 1983.

Dipartimento di Scienze Matematiche, Università di Trieste, Piazzale Europa 1, 34127 TRIESTE, ITALY

Département de Mathématiques, bât. 425, Université de Paris-Sud, 91405 Orsay Cedex, France 